
Resilience in Teams and Organizations

7

8

9

10

11

12

13

14

15

16

17

18

19

20

21

22 Wagstaff, C. R. D., Fasey, K. J., \& Sarkar, M. (2020). Resilience in teams and

23 organizations. In Hackfort, D. \& Schinke, R. J., (Eds.), The Routledge International

24 Encyclopaedia of Sport and Exercise Psychology: Volume 1: Theoretical and

25
Christopher R. D. Wagstaff ${ }^{1}$, Kirsten Fasey $^{2}$, \& Mustafa Sarkar ${ }^{2}$, ${ }^{1}$ University of Portsmouth, UK

${ }^{2}$ Nottingham Trent University, UK 


\section{Resilience in Teams and Organizations}

Over the past couple of decades, our understanding of the pressures faced by those involved in sport has expanded rapidly, with a growing realization that sources of strain in sport are prevalent and pervasive and can originate from a variety of sources (see, e.g., Arnold \& Fletcher, 2012; Gould, Jackson, \& Finch, 1993; Mellalieu, Neil, Hanton, \& Fletcher, 2009; Scanlan, Stein, \& Ravizza, 1991; Thelwell, Weston, \& Greenlees, 2007). These stressors may be associated with an athlete's competitive performance, organizational environment, or personal "non-sporting” life events (Fletcher, Hanton, \& Mellalieu, 2006; see also Sarkar \& Fletcher, 2014). In addition to the growing understanding of these demands it is common for researchers as well as coaches, performance directors, and sports organizations to delineate between athletes, teams, and most recently organizations who thrive under pressure and achieve peak performance and wellbeing and those who yield to pressure and underperform or whose wellbeing suffers at the expense of their success. These differences are often attributed to the concept of resilience with some researchers indicating that resilience is a prerequisite for sporting success (Holt \& Dunn, 2004; Mills, Butt, Maynard, \& Harwood, 2012; Van Yperen, 2009).

Resilience is best understood when it is considered in a context-specific domain (Luthar \& Cicchetti, 2000) and it follows that one should not assume that models of resilience will necessarily be transferable to other domains within or outwith the sport context. Thankfully, a body of work has recently emerged exploring resilience specifically in athletes (Fletcher \& Sarkar, 2012; Galli \& Vealey, 2008; Machida, Irwin, \& Feltz, 2013; Martin-Krumm, Sarrazin, Peterson, \& Famose, 2003; Mummery, Schofield, \& Perry, 2004; Schinke \& Jerome, 2002; Seligman, Nolen-Hoeksema, 
1 Thornton, \& Thornton, 1990), teams (Morgan, Fletcher, \& Sarkar, 2013, 2015, 2019),

2 and organizations. Several reviews of this emerging literature exist (e.g., Fasey, 2017;

3 Fletcher \& Sarkar, 2013; Galli \& Gonzalez, 2015; Linnenluecke, 2017; Morgan,

4 Fletcher, \& Sarkar, 2017; Wagstaff, Sarkar, Davidson, \& Fletcher, 2017). For instance,

5 Fletcher and Sarkar (2013) provided a review and critique the various definitions,

6 concepts and theories of psychological resilience. Galli and Gonzalez (2015) critically

7 reviewed recent conceptual developments in psychological resilience research in sport

8 and highlighted potential areas of future research. More recently, Wagstaff et al. (2017)

9 highlighted the salience, but relative dearth, of systematic examination of the influence

10 of sociocultural factors and context in understanding resilience (cf. Galli \& Vealey,

11 2008; Machida et al., 2013). Taking the last of these reviews and its central observation

12 - the importance but limited presence of sociocultural perspectives on resilience - is somewhat surprising given the growing recognition of the value of such perspectives in non-sport resilience literature (cf. Ungar, 2012). To elaborate, Ungar (2012), argued both "culture and context shape the environment in which processes associated with resilience occur, making some processes more crucial to adaptation and growth than others” (p. 387). Indeed, Wagstaff et al., concurred with this perspective adding, "sociocultural factors and organizational contexts hold significant implications for the definition and development of resilience" (p. 121). In this chapter, we critically review the concept resilience in sport, with a specific emphasis on the sociocultural influences on and the organizational dynamics surrounding resilience. Before considering the emerging team and organizational resilience work, we provide a background to conceptualization of psychological resilience and early research examining this construct in sport. 


\section{Defining Psychological Resilience}

Researchers across various domains of psychology have provided numerous definitions which vary significantly depending on the context of investigation and application, and the conceptualization of resilience as a trait or process (see, for a review, Fletcher \& Sarkar, 2013). Despite resilience being conceived in different ways, researchers generally agree that for this construct to be demonstrated both adversity and positive adaptation must be evident (Fletcher \& Sarkar, 2013). In an attempt to draw on the extant consistencies within the conceptual literature, Fletcher and Sarkar defined psychological resilience as, "the role of mental processes and behavior in promoting personal assets and protecting an individual from the potential negative effect of stressors" (2012, p. 675; 2013, p. 16). The authors proposed this definition extended previous conceptual work in the area by offering a specific focus on psychological resilience, encapsulating aspects of both trait and process conceptualizations, an emphasis on the neutral term "stressor" rather than the negative term "adversity", and a focus on promoting personal assets and protecting an individual from the potential negative effect of stressors rather than positive adaptation per se.

Despite the definitional advances noted above, Wagstaff et al. (2017) noted several additional conceptual considerations regarding the development of socioculturally and contextually sensitive perspectives on resilience. First, the assumption that resilience is a virtue across all contexts leads well-intentioned theory, research, and praxis astray and is germane for resilience to become a vice instead of a virtue in some circumstances. That is, the decontextualization of resilience as a standalone virtue by researchers who characterize it as an individual trait, leave the concept open to misunderstanding and misapplication. A second consideration in the context of 
1 definition resilience relate to the limited acknowledgment of sociological and cultural

2 utility (Fletcher \& Sarkar, 2013) in many extant definitions and conceptualizations of

3 resilience. That is, although psychological resilience is, by definition, centrally focused

4 on intra-individual processes, greater consideration and sensitivity to sociocultural and

5 organizational influences is required to gain a more complete understanding of the

6 phenomenon (Fletcher \& Sarkar, 2013; Sarkar \& Fletcher, 2014). Third, some

7 researchers have problemized the use of the term "resilience" noting that the inherent

8 and perpetual pursuit of resilient soldiers (see McGarry, Walklate, \& Mythen, 2015)

9 casts a dark shadow of hegemonic masculinity, gender-role conflicts, and stigma,

10 resulting in demobilization and reintegration problems for some military veterans.

11 Psychological Resilience Research in Sport

Early studies examining resilience in sport performers largely centered on the

role of resilience as a dependent variable in the stress-injury relationship or individual's explanatory style. For instance, Smith, Smoll, and Ptacek (1990) examined the ways in which moderator variables interact with one another to increase vulnerability or resilience in the life stress-athletic injury relationship. Smith et al. interpreted their data to indicate that social support and psychological coping skills were statistically independent psychosocial resources and operated in a conjunctive manner to influence the relationship between life stress and subsequent athletic injury in adolescents. Adopting an explanatory style perspective on resilience, Seligman et al. (1990) found university swimmers with an optimistic explanatory style to swim faster in subsequent trials following false negative performance feedback compared to their own original time. This work was later extended by Martin-Krumm et al. (2003) who manipulated the beliefs of high school students by telling them that they had not performed as well in 
1 a basketball task in comparison to others. In findings that were similar to those of

2 Seligman et al. (1990), participants with an optimistic explanatory style performed

3 better on the second trial than comparable participants with a pessimistic outlook. In

4 Martin-Krumm et al.'s study, the relationship between explanatory style and

5 participants' dribbling performance after perceived failure was also affected by their

6 anxiety levels and success expectations. Here, an optimistic explanatory style correlated

7 with expectations of successful performance prior to the second trial and lower state

8 anxiety which, in turn, were also linked to improved performance in the dribbling task.

$9 \quad$ Mummery et al. (2004) sought to improve the ecological validity of the then

10 nascent findings by exploring resilience in swimmers who were competing in a real

national competition. Swimmers were classified as resilient if they were able to improve their qualifying time after initially failing to do so during an earlier round. The results showed that athletes classified as resilient had higher perceptions of physical endurance, indicating a more optimistic outlook. Interestingly, the results also demonstrated that these swimmers had lower levels of social support than those who did not perform well following initial failure. The authors explained these results by stating that the swimmers who displayed resilience may have been able to act in a more independent manner in unfamiliar surroundings than their non-resilient counterparts. In particular, placing less emphasis on requiring social support to achieve sporting success. Yet, it is important to note that the swimmers may not have perceived the inability to match their qualifying time as a stressor. This is because it is common practice for swimmers to limit their performance in earlier heats to preserve energy for later, more challenging, races where optimum performance is required. Based on these studies, Schinke and colleagues (e.g., Schinke \& Jerome, 2002; Schinke, Petersen, \& Couture, 2004) created 
1 the first sport resiliency training program primarily focused on developing optimism

2 skills and were deemed relatively successful by the authors in enhancing resilience in

3 athletes and teams. Despite these positive outcomes, it is important to note that there are

4 several limitations allied with the early interventions and the research on which it was

5 based. For example, by focusing solely on optimism and its role in enabling athletes to

6 overcome setbacks, these interventions elided other factors which play a role in athletes'

7 resilience (see Galli \& Gonzalez, 2015; Sarkar \& Fletcher, 2014). In addition, the

8 studies also exclusively explored the role of explanatory style in overcoming the

9 stressor of failure. This approach restricts our practical understanding of how applicable

10 an optimistic style is to athletes encountering other demands such as organizational

11 stressors (Hanton, Wagstaff, \& Fletcher., 2012). In addition, the research reviewed in this section generally establishes resilience based on the criteria of winning or increased performance such as swimming faster. From a sociocultural perspective, such criteria have limitations since they do not acknowledge the individual goals of the performer or characterize what success is for stakeholders, teams or organizations (cf. Ungar, 2008). Finally, by focusing on resilience from a trait perspective, the early studies reviewed in this section failed to capture the person-environment interactions characteristic of contemporary conceptualizations of resilience as a dynamic process (Fletcher \& Sarkar, 2013; Windle, 2011). The literature outlined above provided an important foundation for the work conducted over the last decade, during which time researchers have adopted a more holistic approach to the conceptualization of resilience and incorporated the use of qualitative designs. The first study to investigate resilience in this way was by Galli and Vealey (2008) who explored athletes' perceptions of resilience in relation to the most 
1 difficult adversity they had encountered. The authors' used their findings to propose a

2 framework highlighting that, following adversity, athletes experienced agitation (e.g.,

3 the use of a variety of coping strategies). In turn, this process resulted in positive

4 outcomes including increased learning, motivation, and perspective. Further, the authors

5 also remarked that these positive outcomes were in part a result of pre-existing

6 sociocultural influences and personal resources. In an attempt to build on Galli and

7 Vealey’s (2008) work, several groups of researchers (e.g., Fletcher \& Sarkar, 2012;

8 White \& Bennie, 2015) have employed inductive qualitative designs to explore

9 resilience in sport. For example, Fletcher and Sarkar (2012) developed a grounded

10 theory of psychological resilience in Olympic champions. Their findings revealed that

11 numerous psychological factors (relating to a positive personality, motivation, confidence, focus, and perceived social support) protected the world's best athletes from the potential negative effect of stressors by influencing their challenge appraisal and meta-cognitions. These constructive cognitive reactions promoted facilitative responses that led to the realization of optimal sport performance. In another qualitative study, to clarify how sport might cultivate resilience, White and Bennie (2015) explored gymnast and coach perceptions about the development of resilience through gymnastics participation. Data analysis revealed that aspects of the gymnastics environment created stress and exposed gymnasts to many challenges in training and competition. Further, features of the sport environment, such as interpersonal relationships and positive coach behaviors, supported gymnasts through these challenges and encouraged them to overcome failure, and gymnastics participation was perceived to develop resilience, life skills, self-efficacy, and self-esteem. In addition to these exploratory studies, researchers have recently turned their attention to psychometric issues and the use of 
1 questionnaires to examine resilience or moderate its relationship with dependent

2 variables (e.g., Gucciardi, Jackson, Coulter, \& Mallett; 2011; Sarkar \& Fletcher, 2013)

3 and considered the predictive role of resilience on well-being (see, e.g., Lu et al., 2016;

4 Vitali, Bortololi, Bertinato, Robazza, \& Schena, 2015).

\section{Social and Organizational Influences on Resilience}

6 As alluded to earlier in this chapter, research examining resilience has been

7 critiqued for being too focused on individual capacities (see Ungar et al., 2008).

8 Although some scholars have pointed to the potential salience of sociocultural factors

9 (e.g., Galli \& Vealey, 2008), most of the resilience research conducted in sport has

10 focused on athletes' psychological processes hitherto eliding the sociocultural context

11 within which this process occurs (Sarkar \& Fletcher, 2014). It follows that research

which aims to address this omission is essential to the development of effective

resilience interventions. The argument for such research endeavors is further supported by the identification of social and cultural factors that influence resilience in non-sport domains (see, e.g., Clauss-Ehlers, 2008) and an emerging recognition of social and cultural influences that exist within sport (see, e.g., Blodgett, Schinke, McGannon, \& Fisher, 2014; Schinke \& Hanrahan, 2009; Wagstaff \& Burton-Wylie, 2018). Indeed, Blodgett et al. recently took stock of the growing body of conceptual research aligned with the cultural sport psychology (CSP; see Schinke \& Hanrahan, 2009) and organizational sport psychology (OSP; Fletcher \& Wagstaff, 2009; Wagstaff, 2017; 2019a; 2019b) agendas. Indeed, cultural praxis is central to CSP and OSP as a means of overcoming the 'taken for granted' way of 'doing' sport psychology steeped in a postpositivist, white, Euro-American, male, performance-based discourse, and is pivotal for the future advancement of a socioculturally and contextually sensitive approach to the 
study and development of resilience.

Cultural praxis in sport psychology grew out of early writings that drew on cultural studies that highlighted how issues of power and privilege were being perpetuated in and through the practices of the domain. Through cultural praxis, researchers and practitioners strive to consider their own, as well as others', cultural identities. The intent is to draw attention to issues of sociocultural difference, power, ethics, and politics, which are often concealed, and facilitate a more contextualized understanding of marginalized identities and a plurality of differences (e.g., race, ethnicity, class, gender, sexuality, dis/ability, physicality, nationality). For example, athletes originate from a diverse range of family backgrounds with varying cultural and religious beliefs, factors which have been found to influence the resilience process (cf. Clauss-Ehlers, 2008). Athletes also operate within organizational environments that have similar, yet idiosyncratic, economic, political, and sociocultural characteristics (Fletcher \& Wagstaff, 2009). In light of these influences, it would appear that "off-theshelf" resilience interventions are unlikely to be effective across all athletes, teams, and sport organizations, and there is a need to better incorporate lessons from sociocultural and organizational dynamics research.

Within the general psychology literature, Ungar and colleagues (Ungar, 2008; Ungar et al., 2008; Ungar, 2010; Theron et al., 2011) have written widely about the need for a more culturally and contextually embedded understanding of resilience. In doing so, these authors have used the tenets of ecological theory to draw from research and clinical experience with children, youth, and families to argue that resilience is not a phenomenon solely related to the individual, but also exists as a facet of one's social and political setting, thus being negotiated by individuals and their community. For 
1 other scholars (e.g., Gilligan, 2004; Seccombe, 2002), an approach to resilience

2 development wherein 'changing the odds' is preferable to resourcing individuals to

3 'beating the odds'. To illustrate, in his manual for child and youth care workers,

4 Gilligan (2004) argued that "resilience... is now more usefully considered as a variable

5 quality that derives from a process of repeated interactions between a person and

6 favorable features of the surrounding context in a person's life" (p. 94). Hence, and in

7 line with a more culturally and contextually embedded view of resilience, "the degree of

8 resilience displayed by a person in a certain context may be said to be related to the

9 extent to which that context has elements that nurture this resilience" (p. 94).

Ungar (2012) highlighted the influence of three main sociocultural influences relevant for resilience. The first centers on the observation that facilitative environments can be more powerful than individual-level variables in the resilience process (see Ungar, 2012). To illustrate, Chauhan, Reppucci, Burnette, and Reine (2010) found that, when matching for individual factors such as delinquency and psychological risk factors, the recidivism rates amongst girls were shown to be correlated with sociocultural factors and racial background. In the context of sport, Galli and Vealey (2008) noted that the majority of African American athletes in their study believed that the notion of success and overcoming challenges was a central part of their culture and a key influence on their ability to deal with the adverse events they encountered. In practical terms, these studies underline the need for researchers to not only focus interventions on athletes' personal qualities, but also to utilize aspects of their sociocultural environment to facilitate the development of resilience. Ungar's (2012) second observation concerns the access to, and the meaningfulness of, the findings from non-diverse samples. Policymakers in sport need 
1 to develop organizations and services that facilitate the development of resilience whilst

2 being considerate of different contexts and cultures. Beyond sport, resilience researchers

3 in general psychology have acknowledged that minority groups (Ungar, 2008) and

4 disadvantaged individuals (Hutcheon \& Lashewicz, 2014) are frequently not included in

5 discussions when designing services to aid their resilience. Accordingly, the

6 aforementioned researchers recognized that interventions that target these populations

7 are often not specific to their backgrounds or properly suited to their specific needs.

8 This omission can have dramatic (negative) effects, as highlighted by Hansson and

9 colleagues (Hansson, Tuck, Lurie, \& McKenzie, 2012), who found that adult

10 immigrants who assimilate into a culture and lack specific cultural support report more

11 mental health issues than those who do not. Hence, and given the global nature of contemporary sport, it is vital that practitioners work with athletes to ensure that resources are tailored to the specific sociocultural context in which they originate or identify with. Only then are resilience interventions likely to be both effective and efficacious.

The last observation made by Ungar (2012) was that individuals' benefit more from protective factors developed to alleviate risks when the level of exposure to the risk is at its greatest. Indeed, it is generally accepted that individuals with the greatest perceived needs benefit from unique individual provision rather than general resilience programs designed for wider populations and situations (cf. Robertson, Cooper, Sarkar, \& Curran, 2015). In addition, the support required can be disproportionately larger for an athlete facing substantial demands. Away from sport, education studies have shown smaller class sizes and a caring teacher are more advantageous to pupils with the most complex educational needs and disrupted home lives (Shernoff \& Schmidt, 2008). 
1 Despite the different domains, the overall picture is that a homogenous approach to

2 developing resilience is not appropriate and it is possible that athletes who are most at

3 risk will benefit the most from resilience intervention resources.

$4 \quad$ Team Resilience in Sport

5

Resilience researchers, in various domains of psychology, have recently devoted attention to the group level (e.g., Carmeli, Friedman, \& Tishler, 2013; Stephens, Heaphy, Carmeli, Spreitzer, \& Dutton, 2013). Within the sport psychology literature, Morgan, Fletcher, and Sarkar (2013) conducted the first study of team resilience in sport. Employing focus groups with members of five elite sport teams, a definition of team resilience was developed and the resilient characteristics of elite sport teams were identified. Specifically, team resilience was defined as a "dynamic, psychosocial process which protects a group of individuals from the potential negative effect of the stressors they collectively encounter. It comprises of processes whereby team members use their individual and collective resources to positively adapt when experiencing adversity" (p. 552). Team resilience was described as a dynamic phenomenon with participants stating that was "dependent upon what time of the season it is" or "whether there is an injury in the team". In terms of its protective function, the participants described team resilience as akin to "having a barrier around you" and "having a thick skin". Furthermore, the participants emphasized that team resilience involved a shared experience of stressors (e.g., team disruptions, low team morale) and this was revealed through comments such as "we have been through so many setbacks together". Four resilient characteristics of elite sport teams emerged from this study: group structure (i.e., conventions that shape group norms and values), mastery approaches (i.e., shared attitudes and behaviors that promote an emphasis on team improvement), social capital 
1 (i.e., the existence of high quality interactions and caring relationships within the team),

2 and collective efficacy (i.e., the team's shared beliefs in its ability to perform a task).

The recent developments in resilience research have advanced psychologists'

4 knowledge of the nature, meaning, and scope of team resilience. In the sport psychology

5 literature, Morgan et al.'s (2013) study provided greater definitional clarity on resilience

6 at the team level (i.e., what team resilience is) and a framework to profile the resilient

7 characteristics of elite sport teams (i.e., what resilient teams 'look' like). Although such

8 knowledge provided descriptive information about the factors that enable teams to

9 withstand stressors, these characteristics do not explain how resilient teams function.

10 Morgan et al. described team resilience as a "dynamic, psychosocial process" (p. 552)

11 which points to operational aspects of this construct and how it changes over time. They

12 went on to argue that "due to the contextual and temporal nature of team resilience,

13 future studies should aim to identify the processes that underpin the resilience

14 characteristics" (p. 558). In an attempt to address this gap in our knowledge, Morgan,

15 Fletcher, and Sarkar (2015) subsequently explored the psychosocial processes

16 underpinning team resilience in elite sport. Using narrative inquiry, Morgan et al.

17 (2015) analyzed the autobiographies of eight members of the 2003 England rugby union

18 World Cup winning team. Findings revealed five main psychosocial processes

19 underpinning team resilience: transformational leadership, shared team leadership, team

20 learning, social identity, and positive emotions. The results indicated that these

21 processes enabled the England rugby team to effectively utilize their cognitive,

22 affective, and relational resources to act as leverage points for team resilience when

23 facing stressors. Further, the findings of this study revealed that team resilience was

24 illuminated through a progressive narrative form. This was portrayed by team members 
evaluating stressors in a positive fashion and focusing on moving forward as a team despite setbacks.

Research investigating team resilience in elite sport has begun to describe what resilient teams 'look like' (i.e., their characteristics) and how they function (i.e., their processes). However, less is known about the psychosocial enablers and cues that stimulate such mechanisms and the associated pathways to team resilience (Morgan et al., 2017; Wagstaff et al., 2017). In their discussion of future research directions, Morgan et al. (2015) proposed that "creative qualitative approaches such as ethnography offer intriguing possibilities to study 'first-hand' the underlying team resilience mechanisms ... and how they are developed (p. 76). Thus, through prolonged fieldwork, Morgan, Fletcher, and Sarkar (2019) conducted a season-long (11 months) ethnography to explore the psychosocial enablers and strategies that promote the development of team resilience within a high-level sports team. The sample consisted of a leading English national league-winning semi-professional rugby union team $(n=27)$ participants. Multiple data collection methods were employed (i.e., observation, interviewing, field notes, reflexive diary) as part of a holistic ethnographic approach. An iterative process of content data analysis was employed to identify key themes. Findings revealed five categories comprising multiple practical strategies, actions, and enablers for team resilience development: Inspiring, motivating, and challenging team members to achieve performance excellence; developing a team regulatory system based on ownership and responsibility; cultivating a team identity and togetherness based on a selfless culture; exposing the team to challenging training and unexpected/difficult situations; and promoting enjoyment and keeping a positive outlook during stressors. The findings of this study provide sport psychologists, coaches, and those working in 
teams with multiple psychosocial enablers and strategies to develop team resilience. Collectively, the emerging research exploring team resilience in sport has contributed to our understanding of what team resilience is, highlighted some of the processes by which resilient teams function, and identified evidence-based practical strategies to improve team resilience. Nevertheless, there remains much to be explored regarding the interplay of sociocultural and organizational dynamics and both individual and team resilience. Indeed, some of the themes to emerge from Morgan et al.'s (2013) study of team resilience (e.g., psychosocial conventions shaping group norms and roles, managing change, and social capital) intersect with those highlighted in research on organizational functioning in sport (cf. Wagstaff, Fletcher, \& Hanton, 2012a).

\section{Organizational Resilience}

As alluded to earlier in this chapter, recent resilience research has shifted from individuals toward the study of groups and teams (see, e.g., Alliger et al., 2015; Bennett, Aden, Broome, Mitchell, \& Rigdon, 2010; Meneghel, Salanova, \& Martinez, in press; Morgan et al., 2013; 2015; Stephens et al., 2013). Over the past couple of decades, the concept of resilience has also been applied to organizations (e.g., Gittell, Cameron, Lim, \& Rivas, 2006; Lengnick-Hall, Beck, \& Lengnick-Hall, 2011; McManus, Seville, Vargo, \& Brunsdon, 2008). This work has been shaped not only by the individual and team resilience literature, but also with dominant influences from ecological and engineering resilience, and from disaster management and business continuity research (Annarelli \& Nonino, 2016), where the focus is often on the resilience of a system, rather than a single individual or team (van der Vegt, Essens, Wahlström, \& George, 2015). Within this context, organizational resilience is seen as an emergent property of a complex system with multiple interacting parts which includes the employees and teams 
working within that organization.

Organizational resilience has been defined as, "the maintenance of positive adjustment under challenging circumstances such that the organization emerges from those conditions strengthened and more resourceful" (Vogus \& Sutcliffe, 2007, p. 3418) and "having the capacity to change before the case for change becomes desperately obvious" (Hamel \& Valikangas, 2003, p. 54). Nevertheless, definitions and operationalizations of the concept remain quite fragmented (Tarba, Cooper, Ahammad, Khan, \& Rao-Nickolson, 2017), in part due to the heterogeneity of research streams within this literature (Linnenluecke, 2017). Despite this fragmented and siloed work, reviews of the organizational resilience literature have recently emerged (e.g., Annarelli \& Nonino, 2016; Fasey, 2017; Linnenluecke, 2017).

Linnenluecke's (2017) review charts the historical development of organizational resilience research streams, with the earliest work (e.g., Meyer, 1982) considering organizational resilience as a response to external threats. This history is intertwined with societal events, for instance, a number of industrial accidents in the 1980s including Chernobyl, Exxon Valdez, and the Space Shuttle Challenger prompted an inward-facing focus on resilience as reliability (e.g., Weick \& Roberts, 1993; Boin \& van Eaten, 2013). Additionally, organizational resilience work has also been linked to key external threats (e.g. Gittell et al., 2006; McManus et al., 2008), prompted by high(9/11 and the 2008-09 global financial crisis. Moreover, organizational resilience research has increasingly been influenced by concerns regarding the generalizability of the "reliability" research conducted in larger organizations to smaller businesses (Sullivan-Taylor \& Branicki, 2011). This research, influenced by the contemporaneous development of management literature on business continuity and crisis management, 
1 can be divided into three principle streams, namely those focusing on organizational

2 resilience as a function of employee strengths (e.g., Coutu, 2002; Lengnick-Hall et al.,

3 2011), organizational resilience as the adaptability of the wider system (e.g. Vogus \&

4 Sutcliffe, 2007; Hamel \& Valikangas, 2003), and organizational resilience in relation to

5 inter-organizational networks of supply chains (e.g., Sheffi \& Rice, 2005).

$6 \quad$ Starting with the seminal research by Meyer (1982), whose empirical study

7 explored hospital responses to an unexpected doctors' strike, analyzing financial

8 records, occupancy figures and payrolls across 19 hospitals in addition to a more

9 intensive study of three hospitals with maximally disparate strategies. Meyer suggested

10 that organizational adaptations are divisible into three phases: anticipatory, responsive,

11 and readjustment, and that organizations display two different forms of adaptability, either by absorbing the impact of events or undergoing change and learning. Predictors of adaptation included entrepreneurial strategies and adaptive ideologies which enhance learning, whereas formalized and complex structures were found to retard learning. Following Meyer's seminal work, a second phase of research emerged, focusing on minimizing internal threats by pursuing 'reliability' over efficiency was influenced by the work conducted by a body of researchers at the University of California at Berkeley studying organizations managing hazardous essential technical systems, labelled 'high reliability organizations' (e.g. Roberts, 1989; Bourrier, 2011). Against this backrop, Weick and Roberts (1993) introduced the idea of the 'collective mind' for high reliability, emphasizing the need for cooperation, ongoing interrelating and dense interactions, and the importance of social processes, where individualism was subsumed by the collective mind. The principles were later expanded in the book, 'Managing the Unexpected', in which Weick and Sutcliffe (2001) promulgated five core principles: 
1 monitor small failures; don't oversimplify; be sensitive to the messy reality of what is

2 happening; be committed to resilience; and defer to expertise. 6 Coutu, 2002; Lengnick-Hall et al., 2011) the focus was on human resources as the most A third phase of research outlined by Linnenluecke (2017) was dedicated to how organizations of varying sizes cope and respond in conditions of great environmental uncertainty, such as those experienced at the start of the $21^{\text {st }}$ century. For some (e.g., important part of an organizational system, where employee characteristics were seen as a critical source of resilience capacity, and relationship networks determine the accessibility of these resources (van der Vegt et al., 2015). More recently, LengnickHall et al. (2011) proposed that an organization's capacity for resilience is developed through strategically managing human resources to create competencies among core employees, that when aggregated at the organizational level, make it possible for organizations to achieve the ability to respond in a resilient manner when they experience severe shocks. Specifically, their proposition is based on three elements: cognitive factors (e.g., strong core values, sense of purpose, constructive sense-making), behavioral characteristics (e.g., learned resourcefulness, bricolage, behavioral preparedness), and contextual conditions (e.g., psychological safety, deep social capital, diffused power and accountability).

Alongside the work on human resources, an alternative stream of research in response to external threats considered organizational resilience as the adaptability of business models (Linnenluecke, 2017). Influential work by a body of New Zealand researchers has emanated from an early study by McManus et al. (2008), where organizational resilience was characterized as consisting three main dimensions namely situation awareness (viz. an organization's understanding and perception of its entire 
1 operating environment), management of keystone vulnerabilities (viz. those aspects of

2 an organization, operational and managerial, that have the potential to have significant negative impacts in a crisis situation), and adaptive capacity (viz. the culture and dynamics of an organization that allow it to make decisions in a timely and appropriate manner, both in day-to-day business and also in crises). A resilience benchmarking tool was subsequently developed, divided into three main attribute categories of leadership and culture, networks and relationships, and change readiness (Lee, Vargo, \& Seville, 2013; Whitman, Kachali, Roger, Vargo, \& Seville, 2013).

More recently, researchers have started to consider the processes which support the development of organizational resilience. Burnard, Bhamra, and Tsinopoulos (2018) used case study data from three energy sector organizations to propose four ways to build resilience, depending on an organization's abilities for preparation (i.e., the degree to which an organization has a systematic approach to risk management) and adaptation (i.e., the degree to which an organization flexibly allocates resources). Burnard et al. also highlighted the iterative decision-making processes in which events are initially detected, with their likely impact is evaluated to determine whether to implement an existing response or adjust and adopt a new response. This process-based approach is consistent with the view that organizational resilience is not a 'one size fits all' construct, and that organizations seeking to develop their resilience need to consider strategies which are relevant to their orientations towards preparation and adaptation. In addition to the work of Linnenluecke (2017) charting the development of different research streams within the organizational resilience literature, a review by Fasey (2017) provided a synthesis of empirical research concerning how resilience functions within organizations. In doing so, the characteristics an organization has 
1 which might predict or enable organizational resilience were grouped into resources

2 (viz. human, financial, physical, social, informational and intangible resources), capabilities (namely the organization's capacity to deploy resources towards a desired goal), culture (as shared values, beliefs, expectations and practices), and structure (consisting of how employees and teams are grouped and how information is shared).

Relevant characteristics from each grouping are discussed, and areas of disagreement or ambiguity highlighted. The underlying mechanisms through which organizational resilience operates were found to comprise planning, adaptation, learning, relationship networks, and leadership. Finally, key contextual variables were identified, including the size of an organization and the sector in which it operates. Somewhat surprisingly, the sport sector has been relatively slow to undertake research in this domain, particularly given the volatile nature of sport, and the high levels of organizational change (Wagstaff, Gilmore, \& Thelwell, 2016). Parenthetically, empirical research (e.g., Stephenson, Vargo \& Seville, 2010) indicates that organizational resilience may be greater in the health and community domain than other sectors, thus indicating that sport might be a fruitful context to example organizational resilience.. To the best of our knowledge, the only study of organizational resilience in sport was conducted by Wicker, Filo, and Cuskelly (2013), exploring the organizational resilience of community sport clubs in the aftermath of major cyclone and flood events. Using data from a survey of sport clubs $(n=200)$ in Queensland, Australia, the findings showed that clubs predominantly used human and financial resources in their recovery efforts, with organizational resilience having a significant positive effect on the extent of the club's perceived overall recovery alongside the number of members and the use of government grants. Those clubs that typically used outdoor sport facilities exclusively 
1 (viz. equestrian, golf, and motor sports) recovered to a significantly lesser extent than

2 clubs providing other sports. These data can be interpreted to indicate that it may be

3 preferable for organizations to dedicate their efforts on sharing and mobilizing third-

4 party physical resources where possible to reduce susceptibility to external turbulence

5 (such as natural disasters or terrorism), rather than the maintenance of spare capacity to

6 buffer the impact of turbulent events (cf. Gittell et al., 2006). This may be particularly

7 pertinent for sport organizations given the prevalent use of public policy to attempt to

8 maximize utilization of sport facilities (Iversen \& Cuskelly, 2015), and the potential

9 public perception of inefficiency associated with under-utilization of resources provided

10 from public funds (Dalgaard-Nielsen, 2017).

11 Overall, organizational resilience not only influences the positive, optimal

12 functioning of the organization itself, but also has the potential to significantly influence resilience in athletes and teams. The body of knowledge in this area remains at a nascent stage and empirical examination of organizational resilience is rather scant both in general and sport psychology contexts, with researchers facing challenges concerning conceptualization and operationalization. In particular, more research is needed to understand the complex relationships between individual, team, and organizational resilience. Nevertheless, the landscape of elite sport presents an array of complex adaptive systems that offer rich opportunities to examine and influence resilience in individuals and organizations.

\section{Conclusion}

To conclude, we draw on the words of Mahoney and Bergman (2002), who stated that the specific sociocultural conditions in which an individual functions must be considered when examining competence, and that "failing to do so may lead to a view 
1 of positive adaptation as a static phenomenon with relevance to only a minority of

2 persons in select circumstances" (p. 212). Given there is growing evidence pointing to

3 the central role of resilience for sustained sporting success and highlighting this

4 construct as a key area of development for athletes, teams, and organizations, it is

5 reassuring that researchers and practitioners have begun to gain a better practical

6 understanding of resilience at multiple levels of analysis and intervention in line with

7 the praxis of our profession. 


\section{References}

Alliger, G. M., Cerasoli, C. P., Tannenbaum, S. I., \& Vessey, W. B. (2015). Team resilience: How teams flourish under pressure. Organizational Dynamics, 44, $176-184$.

Annarelli, A., \& Nonino, F. (2016). Strategic and operational management of organizational resilience: Current state of research and future directions. Omega, $62,1-18$

Arnold, R., \& Fletcher, D. (2012). A research synthesis and taxonomic classification of the organizational stressors encountered by sport performers. Journal of Sport and Exercise Psychology, 34, 397-429.

Bennett, J. B., Aden, C. A., Broome, K., Mitchell, K., \& Rigdon, W. D. (2010). Team resilience for young restaurant workers: Research-to-practice adaptation and assessment. Journal of Occupational Health Psychology, 15, 223-236.

Blodgett, A. T., Schinke, R. J., McGannon, K. R., \& Fisher, L. A. (2014). Cultural sport psychology research: conceptions, evolutions, and forecasts. International Review of Sport and Exercise Psychology, 8, 24-43.

Boin, A., \& van Eeten, M. J. G. (2013). The resilient organization. Public Management Review, 15(3), 429-445.

Bonanno, G. A. (2004). Loss, trauma and human resilience: Have we underestimated the human capacity to thrive after extremely aversive events? American Psychologist, 59, 20-28.

Bourrier, M. (2011). The legacy of the high reliability organization project. Journal of Contingencies and Crisis Management, 19(1), 9-13.

Burnard, K., Bhamra, R., \& Tsinopoulos, C. (2018). Building organizational resilience: 
$1 \quad$ Four configurations. IEEE Transactions on Engineering Management, 1-12.

2 Carmeli, A., Friedman, Y., \& Tishler, A. (2013). Cultivating a resilient top management team: The importance of relational connections and strategic decision comprehensiveness. Safety Science, 51(1), 148-159.

Chauhan, P., Reppucci, N. D., Burnette, M., \& Reiner, S. (2010). Race, neighborhood disadvantage, and antisocial behavior among female juvenile offenders. American Journal of Community Psychology, 38(4), 532-540.

Clauss-Ehlers, C. S. (2008). Sociocultural factors, resilience, and coping: Support for a culturally sensitive measure of resilience. Journal of Applied Developmental Psychology, 29(3), 197-212.

Coutu, D. L. (2002). How resilience works. Harvard Business Review, 80, 46-55.

Fasey, K. (2017). A systematic review of organizational resilience research (Unpublished master's thesis). Loughborough University, Loughborough, UK.

Fletcher, D., Hanton, S., \& Mellalieu, S. D. (2006). An organizational stress review: Conceptual and theoretical issues in competitive sport. In S. Hanton, \& S. D. Mellalieu (Eds.), Literature reviews in sport psychology (pp. 321-374). Hauppauge, NY: Nova Science.

Fletcher, D., \& Sarkar, M. (2012). A grounded theory of psychological resilience in Olympic champions. Psychology of Sport and Exercise,, 13(5), 669-678.

Fletcher, D., \& Sarkar, M. (2013). Psychological resilience: A review and critique of definitions, concepts, and theory. European Psychologist, 18(1), 12-23.

Fletcher, D., \& Wagstaff, C. R. D. (2009). Organizational psychology in elite sport: Its emergence, application and future. Psychology of Sport and Exercise, 10, 427434. 
1 Galli, N., \& Gonzalez, S. P. (2015). Psychological resilience in sport: A review of the literature and implications for research and practice. International Journal of Sport and Exercise Psychology, 3, 243-257.

Galli, N., \& Reel, J. J. (2012). "It was hard, but it was good”: Exploring stress-related growth in Division I athletes. Qualitative Research in Sport, Exercise, and Health, 4, 297-319.

Galli, N., \& Vealey, R. S. (2008). “Bouncing back” from adversity: Athletes' experiences of resilience. Sport Psychologist, 22, 316-335.

Gilligan, R. (2004). Promoting resilience in child and family social work: Issues for social work practice, education and policy. Social Work Education, 23, 93-104.

Gittell, J. H., Cameron, K., Lim, S., \& Rivas, V. (2006). Relationships, layoffs, and organizational resilience. The Journal of Applied Behavioral Science, 42, 300329.

Hamel, G., \& Valikangas, L. (2003). The quest for resilience. Harvard Business Review, 81(9), 52-65.

Hansson, E. K., Tuck, A., Lurie, S., \& McKenzie, K. (2012). Rates of mental illness and suicidality in immigrant, refugee, ethnocultural, and racialized groups in Canada: A review of the literature. Canadian Journal of Psychiatry, 57, 111121.

Hanton, S., Wagstaff, C. R. D., \& Fletcher, D. (2012). Cognitive appraisals of stressors encountered in sport organizations. International Journal of Sport and Exercise Psychology, 10, 276-289.

Holt, N. L., \& Dunn, J. G. (2004). Toward a grounded theory of the psychosocial competencies and environmental conditions associated with soccer success. 
Journal of Applied Sport Psychology, 16, 199-219.

Hutcheon, E., \& Lashewicz, B. (2014). Theorizing resilience: critiquing and unbounding a marginalizing concept. Disability and Society, 29, 1383-1397.

Iversen, E. B., \& Cuskelly, G. (2015). Effects of different policy approaches on sport facility utilisation strategies. Sport Management Review, 18, 529-541.

Lee, A. V., Vargo, J., \& Seville, E. (2013). Developing a tool to measure and compare organizations' resilience. Natural Hazards Review, 14, 29-41.

Lengnick-Hall, C. A., Beck, T .E., \& Lengnick-Hall, M. L. (2011). Developing a capacity for organizational resilience through strategic human resource management. Human Resource Management Review, 21, 243-255.

Linnenluecke, M. K. (2017). Resilience in business and management research: A review of influential publications and a research agenda. International Journal of Management Reviews, 19, 4-30.

Luthar, S. S., \& Cicchetti, D. (2000). The construct of resilience: Implications for interventions and social policies. Development and Psychopathology, 12, 857885.

Machida, M., Irwin, B., \& Feltz, D. (2013). Resilience in competitive athletes with spinal cord injury: The role of sport participation. Qualitative Health Research, $23,1054-1065$.

Mahoney, J. L., \& Bergman, L. R. (2002). Conceptual and methodological considerations in a developmental approach to the study of positive adaptation. Applied Developmental Psychology, 23, 195-217.

Martin-Krumm, C. P., Sarrazin, P. G., Peterson, C., \& Famose, J. (2003). Explanatory style and resilience after sports failure. Personality and Individual Differences, 
35, 1685-1695.

McGarry, R., Walklate, S., \& Mythen, G. (2015). A sociological analysis of military resilience: Opening up the debate. Armed Forces and Society, 41, 352-378.

McManus, S., Seville, E., Vargo, J., \& Brunsdon, D. (2008). Facilitated process for improving organizational resilience. Natural Hazards Review, 9, 81-90.

Meneghel, I., Salanova, M., \& Martínez, I. M. (2016). Feeling good makes us stronger: How team resilience mediates the effect of positive emotions on team performance. Journal of Happiness Studies, 17, 239-255.

Mellalieu, S. D., Neil, R., Hanton, S., \& Fletcher, D. (2009). Competition stress in sport performers: Stressors experienced in the competition environment. Journal of Sports Sciences, 27, 729-744.

Meyer, A. D. (1982). Adapting to environmental jolts. Administrative Science Quarterly, 27, 515-537.

Mills, A., Butt, J., Maynard, I., \& Harwood, C. (2012). Identifying factors perceived to influence the development of elite youth football academy players. Journal of Sports Sciences, 30, 1593-1604.

Morgan, P. B. C., Fletcher, D., \& Sarkar, M. (2013). Defining and characterizing team resilience in elite sport. Psychology of Sport and Exercise, 14, 549-559.

Morgan, P. B. C., Fletcher, D., \& Sarkar, M. (2015). Understanding team resilience in the world's best athletes: A case study of a rugby union World Cup winning team. Psychology of Sport and Exercise, 16, 91-100.

Morgan, P. B. C., Fletcher, D., \& Sarkar, M. (2017). Recent developments in team resilience research in elite sport. Current Opinion in Psychology, 16, 159-164.

Morgan, P. B. C., Fletcher, D., \& Sarkar, M. (2019). Developing team resilience: A 

season-long study of psychosocial enablers and strategies in a high-level sports team. Psychology of Sport and Exercise, 49, 101543.

Mummery, W. K., Schofield, G., \& Perry, C. (2004). Bouncing back: The role of coping style, social support and self-concept in resilience of sport performance. Athletic Insight: The Online Journal of Sport Psychology of Sport and Exercise, 6(3), 1-18. Retrieved from. http://www.athleticinsight.com.

Reid, C., Stewart, E., \& Thorne, G. (2004). Multidisciplinary sport science team in elite sport: Comprehensive servicing or conflict and confusion. The Sport Psychologist, 18, 204-217.

Roberts, K. H. (1989). New challenges in organizational research: High reliability organizations. Industrial Crisis Quarterly, 3, 111-125.

Robertson, I., Cooper, C. L., Sarkar, M., \& Curran, T. (2015). Resilience training in the workplace from 2003-2014: A systematic review. Journal of Occupational and Organizational Psychology, 88, 533-562.

Sarkar, M., \& Fletcher, D. (2013). How should we measure psychological resilience in sport performers? Measurement in Physical Education and Exercise Science, 17, 264-280.

Sarkar, M., \& Fletcher, D. (2014). Psychological resilience in sport performers: A review of stressors and protective factors. Journal of Sports Sciences, 32, 14191434.

Schinke, R. J., \& Hanrahan, S. J. (Eds.). (2009). Cultural sport psychology. Champaign, IL: Human Kinetics.

Schinke, R. J., \& Jerome, W. C. (2002). Understanding and refining the resilience of elite athletes: An intervention strategy. Athletic Insight, 4, 1-13. Retrieved 
from. http://www.athleticinsight.com.

Schinke, R. J., Peterson, C., \& Couture, R. (2004). A protocol for teaching resilience to high performance athletes. Journal of Excellence, 9, 9-18.

Seccombe, K. (2002). "Beating the odds" versus "changing the odds": Poverty, resilience, and family policy. Journal of Marriage and Family, 64, 384-394.

Seligman, M. E., Nolen-Hoeksema, S., Thornton, N., \& Thornton, K. M. (1990). Explanatory style as a mechanism of disappointing athletic performance. Psychological Science, 1, 143-146.

Sheffi, Y., \& Rice Jr, J. B. (2005). A supply chain view of the resilient enterprise. MIT Sloan Management Review, 47, 41-48.

Shernoff, D. J., \& Schmidt, J. A. (2008). Further evidence of an engagementachievement paradox among U.S. high school students. Journal of Youth and Adolescence, 37, 564-580.

Smith, R. E., Smoll, F. L., \& Ptacek, J. T. (1990). Conjunctive moderator variables in vulnerability and resiliency research: life stress, social support and coping skills, and adolescent sport injuries. Journal of Personality and Social Psychology, 58, $360-370$.

Stephens, J. P., Heaphy, E. D., Carmeli, A., Spreitzer, G. M., \& Dutton, J. E. (2013). Relationship quality and virtuousness: Emotional carrying capacity as a source of individual and team resilience. Journal of Applied Behavioral Science, 49, $13-41$.

Stephenson, A., Vargo, J., \& Seville, E. (2010). Measuring and comparing organizational resilience in Auckland. Australian Journal of Emergency Management, 25, 27-32. 
1 Sullivan-Taylor, B., \& Branicki, L. (2011). Creating resilient SMEs: Why one size might not fit all. International Journal of Production Research, 49, 5565-5579.

Tarba, S. Y., Cooper, S. C. L., Ahammad, M. F., Khan, Z., \& Rao-Nickolson, R. (2017), Special issue — call for papers: Resilience in organizations. Applied Psychology, 66, 196-201.

Thelwell, R. C., Weston, N. J. V., \& Greenlees, I. A. (2007). Batting on a sticky wicket: Identifying sources of stress and associated coping strategies for professional cricket batsmen. Psychology of Sport and Exercise, 8, 219-232.

Ungar, M. (2008). Resilience across cultures. British Journal of Social Work, 38, 218 235.

Ungar, M. (2012). Researching and theorizing resilience across cultures and contexts. Preventive Medicine, 55, 387-389.

van der Vegt, G. S., Essens, P., Wahlström, M., \& George, G. (2015). Managing risk and resilience. Academy of Management Journal, 58, 971-980.

Vogus, T. J., \& Sutcliffe, K. M. (2007). Organizational resilience: towards a theory and research agenda, IEEE Systems, Man, and Cybernetics 2007 Conference Proceedings, 3418-3422.

Van Yperen, N. W. (2009). Why Some make it and others do not: Identifying psychological factors that predict career success in professional adult soccer. The Sport Psychologist, 23, 317-329.

Wagstaff, C. R. D. (Ed.) (2017). The organizational psychology of sport: Key issues and practical applications. Abingdon, United Kingdom: Routledge.

Wagstaff, C. R. D. (2019a). Taking stock of organizational psychology in sport: An introduction to the special issue. Journal of Applied Sport Psychology, 31, 1-6. 
1 Wagstaff, C. R. D. (2019b). A commentary and reflections on the field of organizational

2

3

4

5

6

7

8 sport psychology: Epilogue to the special issue. Journal of Applied Sport Psychology, 31, 134-146.

Wagstaff, C. R. D., \& Burton-Wylie, S. (2018). Oranisational culture in sport: A conceptual, methodological and definitional review. Sport \& Exercise Psychology Review, 14, 32-52.

Wagstaff, C. R. D., Gilmore, S., \& Thelwell, R. C. (2016). When the show must go on: Investigating repeated organizational change in elite sport. Journal of Change Management, 16, 38-54.

Weick, K. E., \& Roberts, K. H. (1993). Collective mind in organizations: Heedful interrelating on flight decks. Administrative Science Quarterly, 38, 357-381.

Weick, K. E., \& Sutcliffe, K. M. (2001). Managing the Unexpected. San Francisco, CA: Jossey-Bass.

White, R. L., Bennie, A., \& McKenna, J. (2015). Resilience in youth sport: A qualitative investigation of gymnastics coach and athlete perceptions. International Journal of Sports Science and Coaching, 10, 379-394.

Whitman, Z., Kachali, H., Roger, D., Vargo, J., \& Seville, E. (2013). Short-form version of the Benchmark Resilience Tool (BRT-53). Measuring Business Excellence, 17, 3-14.

Wicker, P., Filo, K., \& Cuskelly, G. (2013). Organizational resilience of community sport clubs impacted by natural disasters. Journal of Sport Management, 27, $510-525$

Windle, G. (2011). What is resilience? A review and concept analysis. Reviews in Clinical Gerontology, 21, 152-169. 